\title{
Developmental competence of in vitro-matured human oocytes obtained from pregnant and non-pregnant women
}

\author{
Pallop Pongsuthirak ${ }^{1}$, Teraporn Vutyavanich ${ }^{2}$
}

'Department of Obstetrics and Gynecology, Buddhachinaraj Hospital Medical School, Phitsanulok; ${ }^{2}$ Division of Reproductive Medicine, Department of Obstetrics and Gynecology, Faculty of Medicine, Chiang Mai University, Chiang Mai, Thailand

Objective: The aim of this study was to compare the rate of maturation, fertilization, and embryo development of in vitro-matured human oocytes derived from pregnant and non-pregnant women.

Methods: Immature oocytes were obtained by needle aspiration from 49 pregnant women (group 1) who underwent a cesarean section at term and 77 non-pregnant women (group 2) who underwent a gynecological operation during the same period (8 months). Healthy immature oocytes (530 in group 1 and 539 in group 2) were cultured and assessed for maturation 36 hours later. Mature oocytes were inseminated by intracytoplasmic sperm injection and cultured up to 144 hours.

Results: The percentage of degenerated oocytes was significantly higher ( $12.1 \%$ vs. $6.3 \% ; p<0.001)$ in group 1 than in group 2 . There was no significant difference in the maturation rate $(66.8 \%$ vs. $68.1 \% ; p=0.698)$, fertilization rate $(66.7 \%$ vs. $67.6 \% ; p=0.857)$, or the rate of formation of good-quality blastocysts ( $46.2 \%$ vs. $47.2 \% ; p=0.898$ ) in oocytes obtained from pregnant and non-pregnant women.

Conclusion: The developmental competence of immature oocytes did not differ between pregnant and non-pregnant women.

Keywords: Competence; Oocyte in vitro maturation; Pregnant women

\section{Introduction}

Conventional oocyte donation involves controlled ovarian stimulation, followed by oocyte retrieval. Both procedures impose additional risks that may not be necessary, making it difficult to find non-commercial donors. A potential alternative source could be immature oocytes obtained during a routine gynecological operation or cesarean section, followed by in vitro maturation (IVM). Previous studies showed

Received: Jun 27, 2018 · Revised: Jul 23, 2018 · Accepted: Sep 7, 2018 Corresponding author: Teraporn Vutyavanich Division of Reproductive Medicine, Department of Obstetrics and Gynecology, Faculty of Medicine, Chiang Mai University, Chiang Mai 50200, Thailand Tel: +66-81-8331237 Fax:+66-53-212716 E-mail:tvutyava@gmail.com

*This study was supported by the University Endowment Fund for Medical Research.

This is an Open Access article distributed under the terms of the Creative Commons Attribution Non-Commercial License (http://creativecommons.org/licenses/by-nc/4.0/) which permits unrestricted non-commercial use, distribution, and reproduction in any medium, provided the original work is properly cited. that this procedure added 10 minutes or less to the operating time and exposed the donors to minimal additional risks [1,2]. The use of such donors would simplify the synchronization of recipients in anticipation for the date of the operation. Collection of immature oocytes during a cesarean delivery is also a promising option for those who are pregnant from a previous in vitro fertilization (IVF) treatment and desire future treatment despite not having any remaining cryopreserved oocytes/embryos [2].

In an animal study, Behboodi et al. [3] found no differences in the maturation, cleavage, blastocyst formation, or hatching rates of blastocysts derived from oocytes of pregnant and non-pregnant cows. In contrast, Gambini et al. [4] reported that pregnant mare oocytes showed a lower maturation rate than those from non-pregnant animals, but found no differences in cleavage or blastocyst development. Conversely, Torner et al. [5] observed more rapid maturation in immature oocytes from non-pregnant camels than in those from pregnant camels. In a primate study, Brzyski et al. [6] obtained a 
good IVM rate for baboon oocytes retrieved during a cesarean operation. Xu et al. [7] obtained similar maturation and developmental rates to the morula stage for baboon oocytes obtained in the proliferative and luteal phases.

In humans, controversy similarly exists regarding the developmental competence of oocytes obtained from pregnant and non-pregnant women. Cha et al. [8] reported the first successful birth from IVM oocytes obtained from a patient with polycystic ovaries. Subsequent reports mainly focused on the successful use of immature oocytes from non-pregnant women [9-12], although there have been a few reports of pregnancies from IVM of oocytes retrieved during cesarean delivery [13-15]. The purpose of this study was, therefore, to compare the maturation, fertilization, and embryo development of human IVM oocytes obtained during cesarean and gynecological operations in a parallel study.

\section{Methods}

This research was conducted at the Department of Obstetrics and Gynecology, Buddhachinaraj Hospital, and supported by the University Endowment Fund for Medical Research. The Research Ethics Committee of the Buddhachinaraj Hospital Medical School approved this study (No. 85/57). All participants provided written informed consent to participate in the study.

\section{Subjects}

Pregnant women who underwent cesarean deliveries at Buddhachinaraj Hospital Medical School were invited to participate in the study if they fulfilled the following inclusion criteria: (1) age 1840 years; (2) a spontaneous singleton pregnancy; (3) a gestational age of 37-40 weeks; and (4) elective repeated or emergency cesarean section not due to fetal distress. Women scheduled for elective gynecological operations were invited to participate in the study if they were 18-40 years old and had not used any hormones for 3 months prior to surgery. In both groups, patients were excluded if they (1) had chronic diseases or medical or obstetric complications that could jeopardize their health by prolonging anesthesia or sur-

Table 1. Characteristics of pregnant and non-pregnant women

\begin{tabular}{lcc}
\hline Characteristics & $\begin{array}{c}\text { Pregnant women } \\
(\mathrm{n}=49)\end{array}$ & $\begin{array}{c}\text { Non-pregnant } \\
\text { women }(\mathrm{n}=77)\end{array}$ \\
\hline Age $(\mathrm{yr})^{\mathrm{a})}$ & $29.9 \pm 5.4$ & $33.4 \pm 5.1$ \\
Gravidity & $1.5 \pm 0.8$ & $1.1 \pm 1.2$ \\
Parity & $0.8 \pm 0.6$ & $0.8 \pm 1.0$ \\
Gestational age $(\mathrm{wk})$ & $38.4 \pm 0.9$ & - \\
Body mass index $\left(\mathrm{kg} / \mathrm{m}^{2}\right)^{\mathrm{a})}$ & $28.0 \pm 4.8$ & $22.7 \pm 4.2$ \\
\hline
\end{tabular}

Values are presented as mean \pm standard deviation.

a) $p<0.001$. gery, (2) could not read, write, or understand the Thai language, or (3) had excessive blood loss or unstable vital signs during the operation.

\section{Oocyte collection}

Cesarean or gynecological operations were performed under general anesthesia or spinal block. All visible follicles were aspirated with a 22-gauge needle connected to a $5-\mathrm{mL}$ syringe, filled with $1 \mathrm{~mL}$ of warmed flushing medium (Ferticult Flushing Medium, FertiPro, Beernem, Belgium) after closure of the uterine wound or before performing gynecological surgery.

\section{In vitro maturation}

Follicular fluid was examined carefully under a stereomicroscope to identify immature oocyte-cumulus complexes (OCCs), which were small and dense. Only healthy-appearing oocytes, with multilayered or sparse cumulus, were used in this study. They were washed with oocyte washing medium, and transferred into SAGE oocyte maturation medium (Cooper Surgical, Trumbull, CT, USA), and cultured in groups of up to three in $25-\mu \mathrm{L}$ drops of maturation medium, supplemented with $75 \mathrm{IU} / \mathrm{L}$ of human menopausal gonadotropin, as recommended by the manufacturer, under paraffin oil at $37^{\circ} \mathrm{C}$ in a humidified atmosphere of $6 \% \mathrm{CO}_{2}$ in air. The oocyte maturation medium was prepared 1 day before use and left to equilibrate overnight in the $6 \% \mathrm{CO}_{2}$ incubator.

Table 2. Details of oocytes harvested from pregnant and non-pregnant women

\begin{tabular}{|c|c|c|c|c|c|}
\hline \multirow{2}{*}{ Variable } & \multirow{2}{*}{$\begin{array}{c}\text { Pregnant } \\
\text { women }\end{array}$} & \multicolumn{3}{|c|}{ Non-pregnant women } & \multirow{2}{*}{$p$-value } \\
\hline & & Follicular & Luteal & Total & \\
\hline Number & 49 & 34 & 43 & 77 & - \\
\hline Oocyte retrieved & 603 & 298 & 277 & 575 & - \\
\hline Oocyte per patient & $12.3 \pm 6.9$ & $8.7 \pm 6.5$ & $6.4 \pm 4.3$ & $7.4 \pm 5.9$ & $<0.001^{\text {a) }}$ \\
\hline $\begin{array}{l}\text { Healthy immature } \\
\text { oocyte retrieved }\end{array}$ & 530 & 282 & 257 & 539 & - \\
\hline $\begin{array}{l}\text { Healthy immature } \\
\text { oocyte per patient }\end{array}$ & $10.8 \pm 6.9$ & $8.2 \pm 6.3$ & $6.0 \pm 4.1$ & $7.0 \pm 5.6$ & $0.001^{\mathrm{b})}$ \\
\hline $\begin{array}{l}\text { Degenerated } \\
\text { oocyte }^{c)}\end{array}$ & $73(12.1)$ & $16(5.4)$ & $20(7.2)$ & $36(6.3)$ & $<0.001^{d)}$ \\
\hline
\end{tabular}

Values are presented as mean \pm standard deviation or number (\%).

${ }^{a}$ Analysis of variance. Post hoc tests showed significant differences between pregnant women and non-pregnant women in the follicular phase ( $p=$ $0.037)$ and in the luteal phase $(p<0.001)$, but not between non-pregnant women in the follicular phase and those in the luteal phase $(p=0.271)$; ${ }^{b)}$ Analysis of variance. Post hoc tests showed a significant difference between pregnant women and non-pregnant women in the luteal phase $(p<0.001)$, but not between pregnant women and non-pregnant women in the follicular phase $(p=0.124)$ or between non-pregnant women in the follicular phase and those in the luteal phase $(p=0.241) ;{ }^{\circ}$ The rate of degenerated oocytes was calculated based on the number of total oocytes retrieved; ${ }^{d)} \mathrm{Chi}-$ square test. 


\section{Fertilization and embryo development}

The maturity of the oocytes was assessed 36 hours post-retrieval, after denudation of their cumulus cells by brief exposure to hyaluronidase. Mature oocytes were inseminated immediately by intracytoplasmic sperm injection (ICSI) to prevent oocyte aging, using spermatozoa donated for research. Following ICSI, oocytes were cultured in groups of up to three in $20-\mu \mathrm{L}$ drops of embryo maintenance medium under paraffin oil. Micro-drops $(25 \mu \mathrm{L})$ of embryo maintenance medium were prepared in $35 \times 10-\mathrm{mm}$ culture dishes (Falcon, Corning Life Sciences, Corning, NY, USA), and left to incubate overnight in a $\mathrm{CO}_{2}$ incubator 1 day before use, as recommended by the manufacturer.

Fertilization was assessed 18 hours after ICSI. After 72 hours of culture, the embryos were transferred into blastocyst medium, and cultured for another 72 hours. Embryos were examined once every 24 hours. Only fully expanded blastocysts and hatching or hatched blastocysts, containing a distinct inner cell mass and trophectoderm layer (grade 322 or better according to the Istanbul Consensus Scoring System) [16], were considered to be good-quality blastocysts.

Table 3. In vitro maturation and developmental competence of immature oocytes from pregnant (group 1) and non-pregnant women (group 2)

\begin{tabular}{|c|c|c|c|}
\hline \multirow{2}{*}{ Variable } & \multicolumn{3}{|c|}{ Source of oocytes } \\
\hline & Group 1 & Group 2 & $p$-value ${ }^{\mathrm{a})}$ \\
\hline Immature oocyte & $530(100)$ & $539(100)$ & - \\
\hline Mature oocyte & $354(66.8)$ & $367(68.1)$ & 0.698 \\
\hline Fertilization & $236(66.7)$ & $248(67.6)$ & 0.857 \\
\hline Cleavage $^{\text {b) }}$ & $212(89.8)$ & $225(90.7)$ & 0.858 \\
\hline Blastocyst (total) $)^{\mathrm{b})}$ & $132(55.9)$ & $137(55.2)$ & 0.935 \\
\hline $\begin{array}{l}\text { Good-quality blastocyst } \\
\text { (expanded blastocyst or beyond) }\end{array}$ & $109(46.2)$ & $117(47.2)$ & 0.898 \\
\hline
\end{tabular}

Values are presented as number (\%).

${ }^{\text {a) }}$ Chi-square test; ${ }^{\text {b) }}$ The cleavage and blastocyst rates were calculated based on the number of fertilized oocytes.
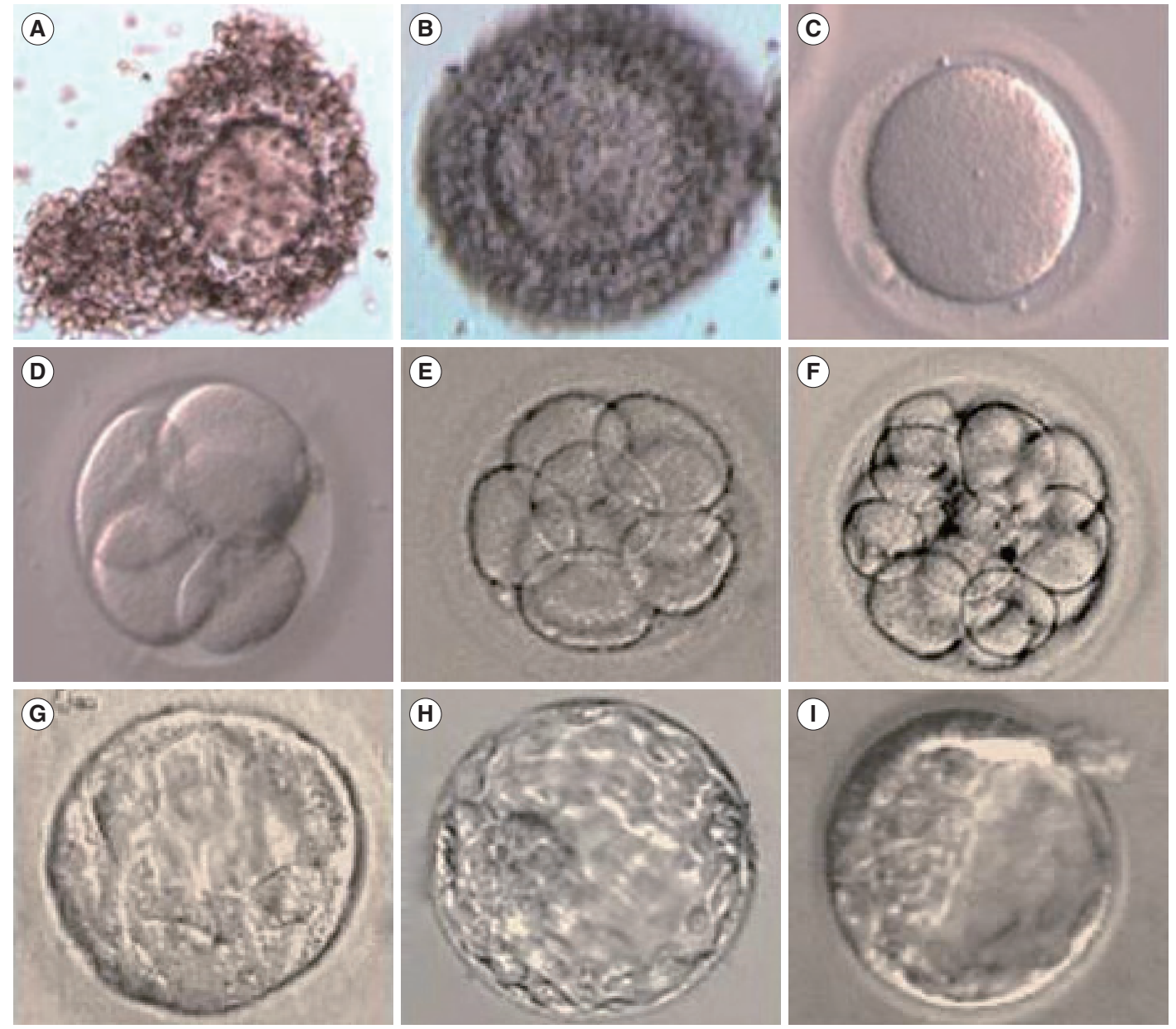

Figure 1. Representative images of oocyte-cumulus complexes, in vitro-matured oocytes, and resulting embryos from pregnant women: (A, B) immature oocytes with multilayered cumulus cells, (C) metaphase II (mature oocytes), (D) four-cell embryo, (E) eight-cell embryo, (F) morula, (G-I) blastocysts. Magnification of all panels, $\times 400$. 


\section{Statistical analysis}

Stata ver. 11 (StataCorp., College Station, TX, USA) was used for all statistical analyses. Comparisons of frequency data between groups, such as fertilization and cleavage rates, were performed using the chi-square test. Means were compared using the non-paired $t$-test or analysis of variance. A p-value below 0.05 was considered to indicate statistical significance.

Given a type I error of 5\% (two-tailed) and a type II error of $20 \%$, sample size calculation showed that at least 400 oocytes were required in each arm to demonstrate a $10 \%$ difference in the maturation rate between oocytes from pregnant and non-pregnant donors.

\section{Results}

Forty-nine pregnant women and 77 gynecological patients volunteered to participate in the study. Their characteristics are shown in Table 1. The indications for surgery were: symptomatic myoma uteri (34 cases), ovarian cyst (12 cases), endometriosis (15 cases), tubal sterilization (11 cases), and polycystic ovary syndrome not responding to clomiphene citrate (five cases). Detailed information about the harvested oocytes is shown in Table 2. The number of follicles per square centimeter at the time of aspiration was found to be significantly higher on the ovarian surface during late pregnancy than during the menstrual cycle ( $7.5 \pm 5.2$ vs. $4.3 \pm 3.5$ follicle $/ \mathrm{cm}^{2}$; non-paired t-test, $p<0.001)$.

A total of 1,069 healthy immature oocytes were included in this study. There was no statistical difference in the IVM rate, fertilization, further cleavage, or blastocyst formation rate among the two groups (Table 3). Representative images of OCCs, IVM oocytes, and resulting embryos from pregnant and non-pregnant women are shown in Figures 1 and 2, respectively.

We collected 282 oocytes from non-pregnant women during the follicular phase and 257 during the luteal phase. There were no significant differences between the groups in the maturation rate, fertilization rate, or rate of formation of good-quality blastocysts (Table 4).

Table 4. In vitro maturation, fertilization, and development of embryos derived from immature oocytes collected during the follicular and luteal phases in non-pregnant women

\begin{tabular}{lccc}
\hline \multirow{2}{*}{ Variable } & \multicolumn{3}{c}{ Source of oocytes } \\
\cline { 2 - 4 } & $\begin{array}{c}\text { Follicular phase } \\
(\mathrm{n}=34)\end{array}$ & $\begin{array}{c}\text { Luteal phase } \\
(\mathrm{n}=43)\end{array}$ & $p$-value ${ }^{\mathrm{a})}$ \\
\hline Immature oocyte & 282 & 257 & - \\
Mature oocyte & $190(67.4)$ & $177(68.9)$ & 0.779 \\
Fertilization & $127(66.8)$ & $121(68.4)$ & 0.842 \\
Cleavage $^{\text {b) }}$ & $113(89.0)$ & $112(92.6)$ & 0.451 \\
Blastocyst & & & \\
Poor-quality & $15(11.8)$ & $14(11.6)$ & 0.952 \\
Good-quality & $58(45.7)$ & $59(48.8)$ & 0.719 \\
\hline
\end{tabular}

Values are presented as number (\%).

${ }^{a}$ Chi-square test; ${ }^{\text {b) }}$ The cleavage and blastocyst rate were calculated based on the number of fertilized oocytes.
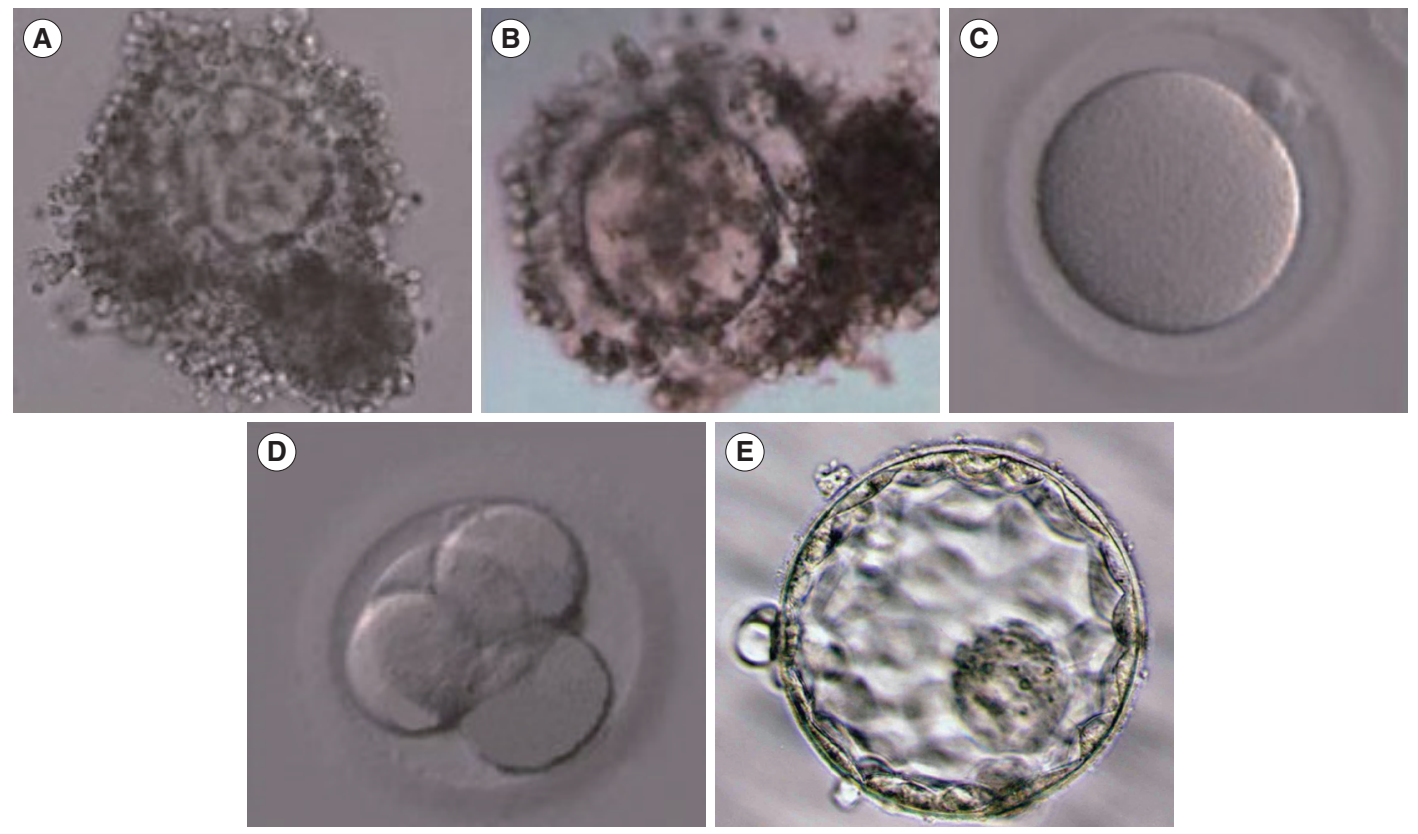

Figure 2. Representative images of oocyte-cumulus complexes, in vitro-matured oocytes, and resulting embryos from non-pregnant women: $(A, B)$ immature oocytes with multilayered cumulus cells, (C) metaphase II (mature oocytes), (D) four-cell embryo, (E) blastocysts. Magnification of all panels, $\times 400$. 


\section{Discussion}

In humans, dominant follicle selection occurs around day 5 of the cycle. The selected follicle continues to develop, while non-selected follicles stop growing and undergo atresia [17]. Although granulosa cells of non-selected follicles show degenerative changes, the oocytes inside are still viable and competent for more than 1 week after follicle selection [18]. This is compatible with our finding that immature oocytes collected at any time before ovulation were developmentally competent and capable of developing into good-quality blastocysts after IVM and fertilization. A recent study confirmed that blastocysts that developed from fertilized IVM oocytes from small non-selected follicles were as competent as those that developed from dominant follicles in terms of pregnancy [18]. The recovery of healthy oocytes in the luteal phase, in this and other studies [19-21], supports the concept that these oocytes came from newly developed follicles that emerged in a wave of luteal recruitment. Based on this current concept, many IVF programs now perform mild stimulation in the follicular phase in poor responders, followed by a second stimulation after oocyte retrieval (double- or dual-stimulation). This method takes advantage of follicular wave emergence in both phases of the menstrual cycle and enables oocyte accumulation in a shorter time frame [22,23].

Follicular development has also been observed throughout various stages of human pregnancy $[24,25]$. The concentrations of steroid hormones in antral follicles during late gestation were found to be almost the same as those in follicles of similar size from non-pregnant women, except for a significantly higher level of progesterone and a slightly lower level of follicle-stimulating hormone (FSH) [26]. This suggests that the regulatory mechanisms of small follicles could be similar during the menstrual cycle and pregnancy [24-26]. The higher follicle density in pregnant women in our study could have been due to bias, as their average age was lower than that of the non-pregnant women. Moreover, patients who came for gynecological operations for ovarian cysts and endometriosis could have had a lower antral follicle count than pregnant women of the same age. The high percentage of oocyte degeneration in the pregnant group could possibly have been due to prolonged exposure to high progesterone and low FSH levels. These findings should be confirmed and elucidated in future studies.

To date, few reports have investigated IVM of immature oocytes collected during the follicular and luteal phases. One study found that luteal oocytes had a significantly higher maturation rate than those retrieved during the follicular phase [27], while another study found slightly lower maturation rates, but a trend towards a higher fertilization rate [28]. In this study, we obtained comparable yields between follicular- and luteal-phase oocytes, in accordance with an- other recent study [29]. The weakness of our study was that it was a prospective parallel study, not a randomized controlled trial, and the two groups might not have been completely comparable in terms of baseline characteristics.

In conclusion, immature oocytes retrieved from pregnant and nonpregnant women were able to undergo IVM, were fertilized, and gave rise to good-quality embryos in comparable yields. As these oocytes can be collected in any phase of the menstrual cycle without hormonal stimulation, and even during pregnancy, this method has a potential to serve as a promising source of oocytes for donation, fertility preservation, and stem cell research. Further studies are needed to define their implantation potential and future clinical use.

\section{Conflict of interest}

No potential conflict of interest relevant to this article was reported.

\section{Acknowledgments}

The authors would like to thank Buddhachinaraj Hospital Medical School for funding and for providing a professional English reviewer, Mr. Robert Moore, to help edit the manuscript.

\section{References}

1. Pongsuthirak P, Vutyavanich T. Developmental competence of human embryos derived from in vitro maturation of immature oocytes retrieved during cesarean section. J Obstet Gynaecol Res 2014;40:459-64.

2. Pongsuthirak P, Songveeratham S, Vutyavanich T. Comparison of blastocyst and Sage media for in vitro maturation of human immature oocytes. Reprod Sci 2015;22:343-6.

3. Behboodi E, Anderson GB, BonDurant RH. Development of in vitro fertilized oocytes from pregnant and nonpregnant cows in oviductal epithelial and cumulus cell co-culture systems. Theriogenology 1992;38:1077-84.

4. Gambini A, Jarazo J, Karlanian F, De Stefano A, Salamone DF. Effect of collection-maturation interval time and pregnancy status of donor mares on oocyte developmental competence in horse cloning. J Anim Sci 2014;92:561-7.

5. Torner H, Heleil B, Alm H, Ghoneim IM, Srsen V, Kanitz W, et al. Changes in cumulus-oocyte complexes of pregnant and nonpregnant camels (Camelus dromedarius) during maturation in vitro. Theriogenology 2003;60:977-87.

6. Brzyski RG, Leland MM, Eddy CA. In vitro maturation of baboon oocytes retrieved at the time of cesarean section. Fertil Steril 1999;71:1153-6. 
7. Xu M, Fazleabas AT, Shikanov A, Jackson E, Barrett SL, HirshfeldCytron J, et al. In vitro oocyte maturation and preantral follicle culture from the luteal-phase baboon ovary produce mature oocytes. Biol Reprod 2011;84:689-97.

8. Cha KY, Koo JJ, Ko JJ, Choi DH, Han SY, Yoon TK. Pregnancy after in vitro fertilization of human follicular oocytes collected from nonstimulated cycles, their culture in vitro and their transfer in a donor oocyte program. Fertil Steril 1991;55:109-13.

9. Trounson A, Wood C, Kausche A. In vitro maturation and the fertilization and developmental competence of oocytes recovered from untreated polycystic ovarian patients. Fertil Steril 1994;62: 353-62.

10. Barnes FL, Crombie A, Gardner DK, Kausche A, Lacham-Kaplan O, Suikkari AM, et al. Blastocyst development and birth after in-vitro maturation of human primary oocytes, intracytoplasmic sperm injection and assisted hatching. Hum Reprod 1995;10: 3243-7.

11. Chian RC, Buckett WM, Tulandi T, Tan SL. Prospective randomized study of human chorionic gonadotrophin priming before immature oocyte retrieval from unstimulated women with polycystic ovarian syndrome. Hum Reprod 2000;15:165-70.

12. Lin YH, Hwang JL, Huang LW, Mu SC, Seow KM, Chung J, et al. Combination of FSH priming and hCG priming for in-vitro maturation of human oocytes. Hum Reprod 2003;18:1632-6.

13. Hwang JL, Lin YH, Tsai YL. In vitro maturation and fertilization of immature oocytes: a comparative study of fertilization techniques. J Assist Reprod Genet 2000;17:39-43.

14. Hwu YM, Lee RK, Chen CP, Su JT, Chen YW, Lin SP. Development of hatching blastocysts from immature human oocytes following in-vitro maturation and fertilization using a co-culture system. Hum Reprod 1998;13:1916-21.

15. Hwang JL, Lin YH, Tsai YL. Pregnancy after immature oocyte donation and intracytoplasmic sperm injection. Fertil Steril 1997; 68:1139-40.

16. Alpha Scientists in Reproductive Medicine and ESHRE Special Interest Group of Embryology. The Istanbul consensus workshop on embryo assessment: proceedings of an expert meeting. Hum Reprod 2011;26:1270-83.

17. Baerwald AR, Adams GP, Pierson RA. Ovarian antral folliculogenesis during the human menstrual cycle: a review. Hum Reprod Update 2012;18:73-91.

18. Teramoto S, Osada H, Sato Y, Shozu M. Nondominant small follicles are a promising source of mature oocytes in modified natu- ral cycle in vitro fertilization and embryo transfer. Fertil Steril 2016;106:113-8.

19. Demirtas E, Elizur SE, Holzer H, Gidoni Y, Son WY, Chian RC, et al. Immature oocyte retrieval in the luteal phase to preserve fertility in cancer patients. Reprod Biomed Online 2008;17:520-3.

20. Maman E, Meirow D, Brengauz M, Raanani H, Dor J, Hourvitz A. Luteal phase oocyte retrieval and in vitro maturation is an optional procedure for urgent fertility preservation. Fertil Steril 2011;95:64-7.

21. Oktay K, Demirtas E, Son WY, Lostritto K, Chian RC, Tan SL. In vitro maturation of germinal vesicle oocytes recovered after premature luteinizing hormone surge: description of a novel approach to fertility preservation. Fertil Steril 2008;89:228.e19-22.

22. Moffat R, Pirtea P, Gayet V, Wolf JP, Chapron C, de Ziegler D. Dual ovarian stimulation is a new viable option for enhancing the oocyte yield when the time for assisted reproductive technnology is limited. Reprod Biomed Online 2014;29:659-61.

23. Ubaldi FM, Capalbo A, Vaiarelli A, Cimadomo D, Colamaria S, Alviggi $C$, et al. Follicular versus luteal phase ovarian stimulation during the same menstrual cycle (DuoStim) in a reduced ovarian reserve population results in a similar euploid blastocyst formation rate: new insight in ovarian reserve exploitation. Fertil Steril 2016;105:1488-95.e1.

24. Govan AD. The human ovary in early pregnancy. J Endocrinol 1968;40:421-8.

25. Govan AD. Ovarian follicular activity in late pregnancy. J Endocrinol 1970;48:235-41.

26. Westergaard L, McNatty KP, Christensen IJ. Steroid concentrations in fluid from human ovarian antral follicles during pregnancy. J Endocrinol 1985;107:133-6.

27. Cha KY, Do BR, Chi HJ, Yoon TK, Choi DH, Koo JJ, et al. Viability of human follicular oocytes collected from unstimulated ovaries and matured and fertilized in vitro. Reprod Fertil Dev 1992;4: 695-701.

28. Kim M, Hong SJ, Lee JH, Min CK, Hwang KJ, Park RW. Comparison of in vitro maturation media of immature oocytes: the effectiveness of blastocyst culture media. Fertil Steril 2011;95:554-7.

29. Grynberg M, Poulain M, le Parco S, Sifer C, Fanchin R, Frydman N. Similar in vitro maturation rates of oocytes retrieved during the follicular or luteal phase offer flexible options for urgent fertility preservation in breast cancer patients. Hum Reprod 2016;31: 623-9. 